\title{
LARGE-SCALE WATER CLASSIFICATION OF COASTAL AREAS USING AIRBORNE TOPOGRAPHIC LIDAR DATA
}

\author{
Julien Smeeckaert ${ }^{1}$, Clément Mallet $^{2}$, Nicolas David ${ }^{2}$, Nesrine Chehata $^{2,3}$, António Ferraz ${ }^{2,4}$ \\ 1: SHOM, 29228 Brest, Cedex 2 - France \\ 2: IGN, MATIS, 73 avenue de Paris, 94160 Saint-Mandé - France; Université Paris-Est \\ 3: IRD/UMR LISAH El Menzah 4, Tunis - Tunisia \\ EA 4592, Laboratoire G\&E, Université de Bordeaux, Pessac - France \\ 4: INESCC, Instituto de Engenharia de Sistemas e Computadores de Coimbra - Portugal \\ firstname.lastnamedign.fr
}

\begin{abstract}
Accurate Digital Terrain Models (DTM) are inevitable inputs for mapping areas subject to natural hazards. Topographic lidar scanning has become an established technique to characterize the Earth surface: and reconstruct the topography. For flood hazard modeling in coastal areas, the key step before terrain modeling is the discrimination of land and water surfaces within the delivered point clouds. Therefore, instantaneous shoreline, river borders, inland waters can be extracted as a basis for more reliable DTM generation. This paper presents an automatic, efficient, and versatile workflow for land/water classification of airborne topographic lidar data. For that purpose, a classification framework based on Support Vector Machines (SVM) is designed. First, a set of features, based only 3D lidar point coordinates and flightline information, is defined. Then, the SVM learning step is performed on small but well-targeted areas thanks to an automatic region growing strategy. Finally, label probabilities given by the SVM are merged during a probabilistic relaxation step in order to remove pixel-wise misclassification. Results over two large areas show that survey of millions of points are labelled with high accuracy $(>95 \%)$ and that small features of interest are still well classified though we work at low point densities $\left(2-3 \mathrm{pts} / \mathrm{m}^{2}\right)$. Finally, our approach provides a strong basis for further discrimination of coastal land-cover classes and habitats.
\end{abstract}

Index Terms - Airborne lidar, classification, coastal areas, 3D features, SVM, relaxation.

\section{INTRODUCTION}

\subsection{Motivation}

Due to global climate change, coastal areas are particularly at risk. However, their physical characteristics are barely described, especially their accurate topography. For natural hazards, remote sensing data at local scales is not sufficient to set up prevention and protection policies. Using optical images or Synthetic Aperture Radar data allows for a rapid landscape-level overview of coastline changes, and helps to identify areas of concern that will require a more indepth study. For such purposes, the small-footprint airborne laser scanning (ALS or lidar) technology appears attractive because such active technique provides fine scale seamless littoral Digital Terrain Models (DTM) over broad extent coverage. Hundreds of kilometers of shoreline can be surveyed with high spatial resolution. The very high altimetric accuracy provided by topographic lidar sensors has opened the field to very precise issues, that were impossible to deal with before. Countrywide surveys are performed for generating national elevation models, e.g., for proper management of the water defences, such as in the Netherlands or in Canada [1].

In France, Litto3D $R$ is a national program, started in 2005, that aims to create a three-dimensional models of the coastlines. ALS is used to produce a continuous land-sea representation of the coast, and the final outputs are DTMs. In particular, the French Mapping Agency (IGN) owns a topographic lidar, that is used to obtain a geographic representation of the terrestrial coastline. Generating DTM first requires the classification of water areas in delivered $3 \mathrm{D}$ point clouds in order to (1) accurately extract the shoreline, (2) calculate the land DTMs, (3) better merge bathymetric and topographic 3D point clouds, and (4) update the existing nautical charts. Consequently, the aim of this paper is to propose a workflow for coastal water/land classification in topographic ALS datasets, efficient at large scales, and adapted to various landscapes.

\subsection{Related works and contributions}

Water detection in 3D point clouds has been tackled through two main approaches. First, direct water/land interface detection is performed. For seashore extraction, the most common method consists in generating a surface model from the 3D point cloud and making an intersection with a given water level, or simpler with the $0 \mathrm{~m}$ line [2]. This is restricted to the fact that the intersecting water level can only be higher than the water level in the LiDAR dataset. Secondly, standard classifiers are adopted for point-based discrimination, using features extracted from the 3D point clouds. Since height information is not sufficient, additional attributes or data sources are inserted, mainly lidar intensity and point density: the reflection properties of water surfaces for near-infra-red lidar beams are characterized by significant absorption or specular reflection resulting in numerous non-recorded laser echoes. [3] use a fuzzy logic classifier, but even with calibrated lidar intensity, poor discrimination between asphalt and water surfaces is noticed. Orthoimages can thus be inserted in an unsupervised Mean-Shift classifier [4]. More advanced geometrical features are inserted in the Conditional Random Fields framework in [5]. Contextual knowledge is therefore introduced and classification performance is improved. In addition, other land classes can be discriminated. All these approaches perform well in terms of classification accuracies but cannot deal with the large scale and adaptativeness to any landscapes issues. As supervised approaches perform well, a supervised classifier, 
namely Support Vector Machines (SVM), is adopted. Our method, detailed below, presents several relevant contributions:

1. A large-scale and fast classifier of water areas. SVM learning step takes advantage of the great number of available information to capture the diversity of the land and water classes.

2. A fully automatic and parameter-free approach. It only requires as input rough polylines describing the sea-land interface (or 2D coastline database when available).

3. A versatile procedure that allows to process a large variety of landscapes. We can deal both with anthropic or natural areas, while detecting at the same time inland waters. No multi-echo, intensity, full-waveform lidar or multi-spectral optical information is necessary, making our work easily reproducible.

\section{DATASETS}

Two study areas have been retained for our analysis. Both have been acquired with a small-footprint OPTECH 3100 system, with a pulse rate of $100 \mathrm{kHz}$, a nominal point density per strip of $2 \mathrm{pts} / \mathrm{m}^{2}$, resulting in a final point density between $2-4 \mathrm{pts} / \mathrm{m}^{2}$ with a $50 \%$ strip overlap. The input datasets correspond to $1 \mathrm{~km} \times 1 \mathrm{~km}$ tiles: strips have been registered and their respective point clouds merged. The planimetric and altimetric accuracies are inferior to $0.2 \mathrm{~m}$ and $0.3 \mathrm{~m}$, respectively.

Perpignan area corresponds to a surface of $365 \mathrm{~km}^{2}$, with a mean point density of $2.3 \mathrm{pts} / \mathrm{m}^{2}$. It is located in the southern French Mediterranean coast, and covers $50 \mathrm{~km}$ of the Languedoc-Roussillon region seashore. It concentrates a wide set of morphologies and coastal environments: low sandy beaches intersected with rocky capes, and regularly distributed nearshore breakwaters. In this area, setups can reach $1 \mathrm{~m}$ under the added action of waves. For this reason, the area is classified as essential monitoring area, and more than $30 \%$ of the coastline is equipped with protection structures.

Mayotte is a tropical island of the Comoro Archipelago and a French department. Here, the available dataset covers $193 \mathrm{~km}^{2}$, with a point density around $3.2 \mathrm{pts} / \mathrm{m}^{2}$. Such area is highly monitored since many natural hazards exist: flooding, landslide, cyclones, erosion, etc. Furthermore, natural hazards are exacerbated by the urban spreading and the biodiversity endangered by the human pressure, demanding up-to-date land-cover maps.

\section{METHODOLOGY}

\subsection{Overall strategy}

The core of the workflow is designed in a 2D-raster mode in order to provide a fast classification, with a tailored learning step. The processing chain can be divided into three steps.

1. Computation of features of interest. Six attributes are designed for discriminating water areas. The 3D lidar attributes are then interpolated in a $2 \mathrm{D}$ regular grid for large scale processing.

2. Learning procedure. The knowledge of the land/water interface is used to define the training pixels the most likely to belong to each class. However, as this delineation may not be up-to-date or accurate enough, more representative training pixels are automatically selected using spatial reasoning.

3. Land/water classification. A supervised Support Vector Machines (SVM) classifier is run on the pixels of the raster grid. Results were finally regularized using probabilistic relaxation, taking into account the probabilities given by the SVM classifier.

SVM was selected because they are adapted to deal with highdimensional spaces and have shown considerable potential in the supervised classification of remotely sensed data, with very limited training amount. In addition, they outperform standard classification methods, as demonstrated by several general or application-driven comparative studies [6], and in particular with airborne lidar data.

\subsection{Discriminative lidar features and $2 \mathrm{D}$ interpolation}

We have retained three families of features. They are based on (i) the height, (ii) the local point density, and (iii) the local shape of the 3D point neighborhood. To deal with any kind of lidar point cloud, we have consciously discarded the intensity/amplitude echo-based features, i.e., information about the position of the 3D point within the current emitted lidar pulse. As a result, our method applies only to the $3 \mathrm{D}$ point coordinates.

\subsubsection{Feature computation and $2 D$ interpolation}

The size of a $3 \mathrm{D}$ point neighborhood is the single parameter to be tuned in the feature computation stage. Given $d$ the point cloud density and $n$ the minimal number of points needed to calculate robust 3D descriptors, the radius $r$ is defined by $r=(n / \pi d)^{1 / 2}$. In practice, 10 points are sufficient. Thus, $r \in$ [1-1.8 m].

Furthermore, the interpolation of the $3 \mathrm{D}$ point cloud features on a regular $2 \mathrm{D}$ grid allows to better handle the large data volume and high dimensionality of the raw point clouds. The main areas of interest are the land-water interface, and, in particular, shallow waters. The larger the grid cell, the more inaccurate the classification. Therefore, the point cloud is resampled at a resolution of $1 \mathrm{~m}$. The mean value of each feature is selected as final descriptor.

\subsubsection{Feature description}

A first attribute is the height of the lidar point with respect to the geoid, $H_{G}$. Nevertheless, it does not always help to discriminate water points in inland areas. However, near-infrared pulse penetrates very little in water volumes, and therefore the $2 \mathrm{D}$ distribution of the lidar points can be used to detect such surfaces. For wide laser pulse scan angles $\left(>15^{\circ}\right)$, part of the emitted radiation returning from water varies significantly and may not be distinguished from the background noise. Therefore, the point density is much lower in the tails of the swath comparing to inland areas. Several 2D density-based features can be derived. The majority density $\left(D_{m}\right)$ is computed: only lidar points from the strip with the highest number of lidar hits are taken into account. In order to avoid the sensitivity to the strip overlap, we define:

$$
D_{m}=\max _{\text {strip1, strip2 }}\left\{D_{\text {strip } 1}, D_{\text {strip2 }}\right\}=D_{\max } .
$$

Additionally, the density ratio $\left(D_{r}\right)$ is introduced to favor areas acquired with several strips but not equally sampled, which is not the case of water surfaces. $D_{r}$ is computed as follows:

$$
D_{r}=\frac{D_{\max }-D_{\min }}{D_{\max }} \in[0,1]
$$

where $D_{\max }$ and $D_{\min }$ are the point densities corresponding, for each cell of the grid, to the strips with the highest and lowest number of points, respectively. Such a value is close to 0 on water areas. Although noisy values can be found at water strip borders, it is particularly suited for our problem. 
Finally, the 3D distribution of lidar points can also be evaluated through the computation of eigenvalue features [7]. A covariance matrix of the 3D coordinates is computed in a cylindrical vertical neighborhood of fixed radius. Such a matrix provides three eigenvalues $\lambda_{1}, \lambda_{2}$, and $\lambda_{3}$ (in descending order). These attributes allow to discriminate water surfaces, which are both horizontal and planar, from land, even for pixels lying on the ground. Two eigenvaluebased features were computed. The first one was the smallest eigenvalue, $\lambda_{3}$ (called volume afterwards). Indeed, $\lambda_{3} \simeq 0$ for planar elements, $\lambda_{3}>0$ for ground pixels (due to ground microreliefs and surface roughness) and $\lambda_{3}>>0$ for real 3D Earth surfaces. The second feature is the scatter, defined as $\mathcal{S}=\lambda_{3} / \lambda_{1} \in[0,1]$. After all, the feature set is: $\left\{H_{G}, D_{m}, D_{r}, \lambda_{3}, \mathcal{S}\right\}$.

\subsection{Learning procedure}

For non-parametric classifiers such as SVM, only samples lying on the edges of a given class distribution in data space contribute to the analysis. Here, the subset generation is carried out automatically using the scatter and the volume features. Extreme values are representative of land and water classes, respectively. High scatter values correspond to areas with significant vertical scattering such as vegetation and buildings. Lowest volume values indicate flattest areas i.e., water surfaces close to the flightline nadir. Thus, we retrieve pure pixels (hereafter called seeds) by thresholding volume and scatter cumulative distribution functions (computed with 500,000 pixels randomly taken). Gradient is computed on both curves. For the volume (scatter) curve, areas inferior (superior) to the highest gradient value are labelled as water and land seeds, respectively. However, seed distribution is not satisfactory. For water areas, few nadir points are selected whereas they are the most similar to land surfaces, while for land regions, significant spatial heterogeneity exist and heterogeneous training pixels cannot be selected with such method.

Since they are not representative of both classes, they are used to design a more adapted training set. This step is based on the historical coastline (HCL) or a rough land-water interface provided by an end-user. Discrepancies may exist between the dataset and such manually designed border: it cannot be used directly. Consequently, we generate a buffer zone centered on the HCL, to prevent the aggregation of unreliable pixels. A percentage of seed points within such buffer zone is used. Starting with pixels belonging to the HCL, the dilatation is stopped when $40 \%$ of the seed points in both classes are included in the buffer. Then, connected regions are retrieved with a binary region growing procedure, and these regions are labelled as water or land according to the majority of seed points. At last, $1 \%$ of their pixels are randomly kept. Finally, the procedure is iterated for $3 \%$ of the tiles of the area, avoiding close areas ( $10 \mathrm{~km}$ constraint) to favor higher heterogeneity in training sets.

\subsection{Land/water classification}

For Support Vector Machines classification, the standard Gaussian kernel is selected and the SVM hyperparameters are optimized with a simple grid search. However, SVM are limited to pixel-based classification which results in noisy outputs. To overcome such limitation [8], the simplest solution, adopted in this paper, is the filtering approach. For each pixel, a new label is computed with respect to the weighted incoming labels of a given neighborhood. Such a solution offers the advantage of linear growth of the computational cost with the number of pixels. For that purpose, we have adopted the relaxation probabilistic framework [9]. We take into account both neighborhood information and the probabilities of belonging to each class, as provided by libSVM [10]. This is an iterative algorithm in which the probability values for each pixel are updated to make them closer to the probabilities of their neighbours. Thus, we compute the membership energy for both classes and assign the label corresponding to the lowest value. Such energy $E$ for label $l$ and pixel $i$ is computed as follows:

$$
E_{l}(i)=\sum_{j \in V(i)} G_{\sigma}(\|i-j\|) \cdot E_{l}(j) \cdot M_{i, j}\left[l, l_{j}\right] .
$$

$G_{\sigma}(\|i-j\|)$ is the weight of pixel $j$, where $G_{\sigma}$ corresponds to the zero-mean Gaussian density function with variance $\sigma^{2}(\sigma=1$ in our experiments) and $V(i)$ is the vicinity window (here a $5 \times 5$ window). Finally, $M$ is called compatibility matrix since it measures the compatibility between pixel $i$ with label $l$ and pixel $j$ with label $l_{j}$. We have:

$$
M=\left(\begin{array}{cc}
0.8 & 0.2 \\
0.2 & 0.8
\end{array}\right) .
$$

$M$ defines a priori correlations between the probabilities of neighboring points and corresponds to conditional probabilities verifying: $0 \leq M_{i, j}\left[l, l_{j}\right] \leq 1$, and $\sum_{l} M_{i, j}\left[l, l_{j}\right]=1$. The coefficients have been empirically selected so as to enforce spatial homogeneity. However, in order to preserve water/land boundaries, the coefficients out of the matrix diagonal are not equal to 0 .

\section{RESULTS}

The Overall Accuracy (OA) is used as accuracy measure (Table 1). Very satisfactory results are achieved (Figure 1). SVM appears to be efficient classifier for large-scale mapping, even if very simple features are introduced and no contextual knowledge is integrated. Higher accuracies are reported for the Perpignan area. It corresponds to a simpler landscape, with less carved and steep shores, and less complex inland water surfaces than for Mayotte. For both areas, water regions are better discriminated than land points (water: 99.7\%-land: 96.8\% for Perpignan and 98.1\%-95.1\% for Mayotte), due to the higher homogeneity of water point behaviours.

A visual assessment confirms that errors mainly correspond to punctual misclassifications (Figure 2). Isolated pixels with a wrong label are present in both classes, located when similar behaviours exist. In water areas, misclassifications correspond to $3 \mathrm{D}$ points that are close to the strip centers, where higher and regular point densities are reported. Conversely, for land regions, pixels are labelled as water for flat and more irregular sampled surfaces. Besides, even if the workflow has been designed for large-scale discrimination, small features are correctly preserved: rocks, bridges, or ships of 5-10 m length on waters and small water surfaces on land regions. Additionally, smooth transitions between both classes are also well detected. This is the case for sandy beaches or river banks. This shows the relevance of density-based features. Harbours, estuaries, cliffs, complex rocky shorelines, sandy beaches, rivers or inland waters can be all correctly classified (Figure 2). This means that the SVM has been trained with the appropriate data set confirming the effectiveness of the proposed training set enrichment procedure. The main remaining issue is that the classification can locally fail in wetlands areas (marshes and mangroves) i.e., for land surfaces impermeated with water: laser pulses are not backscattered, resulting in lower point densities.

The average computing time for the full workflow is around 30 minutes for $1 \mathrm{~km}^{2}$, for a single-core computer. Our method is ten times faster than a human operator demonstrating its relevance for areawide mapping. 

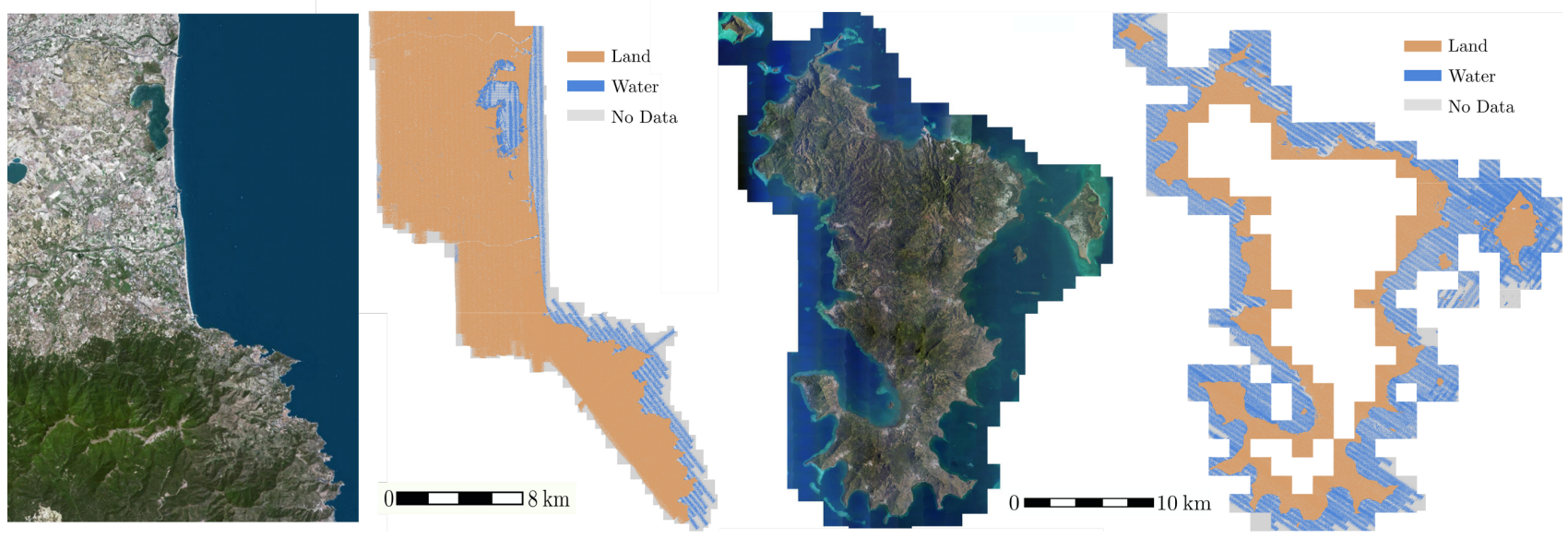

Fig. 1. Results for both study areas. Left: Perpignan. Right: Mayotte. (c) IGN.

\begin{tabular}{|c|c|c|c|}
\hline $\begin{array}{c}\text { Area }(\# \\
\text { pixels })\end{array}$ & After SVM & After relaxation & $\begin{array}{c}\text { Training } \\
\text { set size } \\
(\%)\end{array}$ \\
\cline { 2 - 3 } $\begin{array}{c}\text { Perpignan } \\
(831,814,063)\end{array}$ & 97.47 & OA $(\%)$ & 0.14 \\
\hline $\begin{array}{c}\text { Mayotte } \\
(620,120,967)\end{array}$ & 96.55 & 96.27 & 3.65 \\
\hline
\end{tabular}

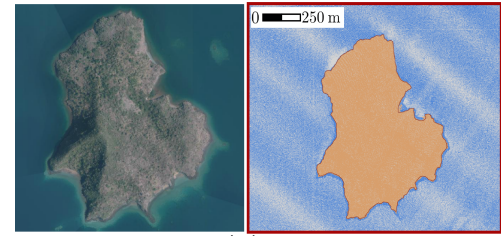

(a)
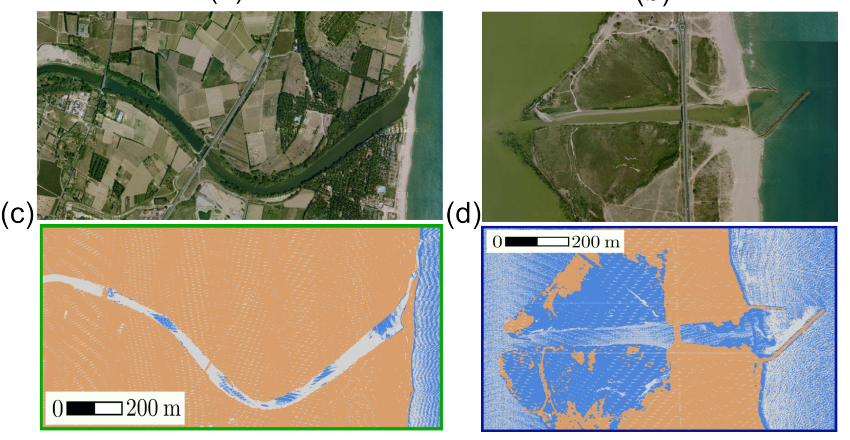

Fig. 2. Results for various areas of interest. (a) Island. (b) Anthropised bay. (c) River. (d) Complex bay mouth and lake. (c) IGN.

[6] G. Mountrakis, J. Im, C. Ogole, "Support vector machines in remote sensing: A review", ISPRS Journal of Photogrammetry and Remote Sensing 66(3), 247-259, 2011.

[7] J. Demantké, C. Mallet, N. David, B. Vallet. Dimensionality based scale selection in 3D lidar point cloud. ISPRS Archives XXXVIII(Part 5/W12), 2011.

[8] K. Schindler, "An overview and comparison of smooth labeling methods for land-cover classication", IEEE Transactions on Geoscience and Remote Sensing 50(11), 4534-4545, 2013.

[9] P. Gong, P. Howarth, "Performance analyses of probabilistic relaxation methods for land-cover classication", Remote Sensing of Environment 30(1), 33-42, 1989.

[10] T. Wu, C.-J. Lin, R. Weng, "Probability estimates for multiclass classication by pairwise coupling", Journal of Machine Learning Research 5, 975-826, 2004.
[5] A. Schmidt, F. Rottensteiner, U. Soergel, "Classification of airborne laser scanning data in Wadden sea areas using conditional random fields," ISPRS Archives XXXIX-B3, 161-166, 2012. 